Ann. Scient. Éc. Norm. Sup.,

4e série, t. 40, 2007, p. 179 à 189.

\title{
DEFORMATION OF HOLOMORPHIC MAPS ONTO THE BLOW-UP OF THE PROJECTIVE PLANE
}

\author{
By JUN-MUK HWANG ${ }^{1}$
}

ABSTRACT. - Let $S$ be the blow-up of the projective plane at $d$ distinct points and $\psi: S^{\prime} \rightarrow S$ be any surjective holomorphic map from a compact complex manifold $S^{\prime}$. We will show that all deformations of $\psi$ come from automorphisms of $S$ if $d \geqslant 3$. The result is optimal in the sense that it is not true if $d \leqslant 2$. The strategy of the proof is to use the infinitesimal automorphisms of the web geometry on $S$ arising from the natural foliations of $S$ induced by the pencils of the lines through the blow-up centers.

(c) 2007 Elsevier Masson SAS

RÉSUMÉ. - Soit $S$ l'éclaté du plan projectif en $d$ points distincts, et $\psi: S^{\prime} \rightarrow S$ une application surjective quelconque d'une variété complexe compacte $S^{\prime}$ dans $S$. Nous montrons que toutes les déformations de $\psi$ proviennent d'automorphismes de $S$ si $d \geqslant 3$. Le résultat est optimal, au sens qu'il n'est pas vrai si $d \leqslant 2$. La stratégie de la preuve est d'utiliser les automorphismes infinitésimaux de la géométrie des tissus sur $S$ provenant des feuilletages naturels de $S$ induits par les pinceaux de droites passant par les points d'éclatements.

(C) 2007 Elsevier Masson SAS

\section{Introduction}

Let $\psi: X^{\prime} \rightarrow X$ be a surjective holomorphic map between two compact complex manifolds. We say that a deformation

$$
\left\{\psi_{t}: X^{\prime} \rightarrow X, \psi_{0}=\psi, t \in \mathbf{C},|t|<\varepsilon\right\}
$$

of $\psi$ comes from automorphisms of $X$ if there exists a holomorphic family of biholomorphic automorphisms $\left\{g_{t},|t|<\varepsilon\right\}$ of $X$ such that $g_{0}=\operatorname{Id}_{X}$ and $\psi_{t}=g_{t} \circ \psi$ for each $t$. In this case, two points of $X^{\prime}$ have the same images under $\psi_{t}$ if and only if they have the same images under $\psi$. This looks like a very strong restriction on the deformation $\psi_{t}$. However, in recent works, it has been discovered that for many target manifolds $X$, all deformations of $\psi$ come from automorphisms of the target. This is the case, for example, when the target is simply connected, algebraic and non-uniruled [7] or a Calabi-Yau manifold [5]. Moreover, in [6,8,9], it was proved that this holds for many Fano manifolds of Picard number 1, excepting the projective space. It is natural to ask what happens when the target is a Fano manifold of Picard number $>1$. In this case, even the 2-dimensional case has not been studied. Our goal is to answer this question for

\footnotetext{
${ }^{1}$ This work was supported by the Korea Research Foundation Grant funded by the Korean Government (MOEHRD) (KRF-2006-341-C00004).
} 
the 2-dimensional Fano manifolds, namely, del Pezzo surfaces. Note that a del Pezzo surface is the blow-up of $\mathbf{P}_{2}$ at $d$ points in general position with $d \leqslant 8$. We will prove the following general result.

MAin TheORem. - Let $S$ be the blow-up of $\mathbf{P}_{2}$ at three or more distinct points. Let $\psi: S^{\prime} \rightarrow S$ be a surjective holomorphic map from a compact complex manifold $S^{\prime}$. Then all deformations of $\psi$ come from automorphisms of $S$. In particular, there is no non-trivial deformation if $S$ has no holomorphic vector field.

In Section 2, we will show that this result is optimal, namely, when $S$ is the blow-up of one or two points, there exist examples of deformations which do not come from automorphisms of $S$.

Our approach to Main Theorem is motivated by the work [9]. The key idea in that work was to use the geometric structure defined by minimal rational curves in the target $X$. When the target is the blow-up of $\mathbf{P}_{2}$, the natural analog of minimal rational curves are those rational curves with trivial normal bundle coming from the pencils of lines through the blow-up centers. But there is an essential difference in our situation from [9]. Unlike in the case of Fano manifold of Picard number 1, the geometric structure induced by deformation of a minimal rational curve on the blow-up of $\mathbf{P}_{2}$ is rather trivial, namely, it is just a fibration. Our idea here is to exploit the nontrivial geometry of the collection of these fibrations, namely, their web geometry.

For this purpose, we will recall in Section 3, the basic notion of web geometry and some computations. However, we need no deep results from the theory of web geometry. All the computations regarding webs in this paper are elementary and use only very basic ordinary differential equations. In fact, while most works in web geometry seem to be centered around the local property, especially, the linearizability question (see [3] for a nice survey), all the webs we encounter here are linear by definition. In this regard, our result shows that even simple linear webs have interesting geometry, related to its infinitesimal automorphisms. Moreover it shows that a certain global aspect of the web geometry, having to do with the monodromy of the infinitesimal automorphisms, can play an important role in problems of algebraic geometry such as our Main Theorem.

In Section 4, we will introduce the notion of webs of fibrations, which is crucial in relating the deformations of holomorphic maps to the infinitesimal automorphisms of webs.

The proof of Main Theorem will be given in Sections 5 and 6. Logically speaking, it suffices to prove Main Theorem when $S$ is the blow-up of $\mathbf{P}_{2}$ at three distinct points. But when it is the blow-up at four or more points (or three collinear points), a stronger result holds, which is an analog of the classical Liouville theorem in conformal geometry $[1,15.2]$. We will treat this case separately in Section 5. The proof for the blow-up at three points will be given in Section 6, using a monodromy argument.

\section{Blow-up at one or two points}

Here we will explain that an analog of Main Theorem for the blow-up of $\mathbf{P}_{2}$ at one or two points cannot hold by giving examples of deformations of surjective holomorphic maps which do not come from automorphisms of the target. Logically, it suffices to do it only for the blowup at two points, since a family of surjective holomorphic maps to the blow-up at two points naturally induces a family of surjective holomorphic maps to the blow-up at one point. However, we will treat the case of the blow-up at one point separately, to show that there are examples which do not factor through the blow-up at two points.

$4^{\mathrm{e}}$ SÉRIE - TOME $40-2007-\mathrm{N}^{\circ} 1$ 
Example 2.1. - Let us start with the blow-up at one point. Choose any surface $M \subset \mathbf{P}_{3}$ and a point $z_{0} \in \mathbf{P}_{3} \backslash M$ to define the projection

$$
f_{0}: M \rightarrow \mathbf{P}_{2}
$$

with the vertex $z_{0}$. Pick $x \in \mathbf{P}_{2}$ such that $f_{0}$ is unramified at

$$
f_{0}^{-1}(x)=\left\{s_{1}, \ldots, s_{r}\right\}
$$

where $r$ is the degree of the surface $M$. Let $\left\{z_{t} \notin M, t \in \mathbf{C},|t|<\varepsilon\right\}$ be a deformation of $z_{0}$ along the line joining $z_{0}$ and $x$. Then the projection with the vertex $z_{t}$ defines

$$
f_{t}: M \rightarrow \mathbf{P}_{2}
$$

which is a deformation of $f_{0}$. If $M$ and $x$ are chosen generically, the deformation $f_{t}$ cannot come from automorphisms of $\mathbf{P}_{2}$. Note that

$$
f_{t}^{-1}(x)=\left\{s_{1}, \ldots, s_{r}\right\}=f_{0}^{-1}(t) \quad \text { for each } t \in \mathbf{C},|t|<\varepsilon .
$$

Let $M^{\prime}$ be the blow-up of $M$ at $s_{1}, \ldots, s_{r}$ and $S$ be the blow-up of $\mathbf{P}_{2}$ at $x$. Then $f_{t}$ induces a surjective holomorphic map $\psi_{t}: M^{\prime} \rightarrow S$. The family $\left\{\psi_{t}, t \in \mathbf{C},|t|<\varepsilon\right\}$ defines a deformation of the holomorphic map $\psi_{0}$ which does not come from automorphisms of $S$.

Example 2.2. - The case of the blow-up at two points is a slight variation of Example 2.1. Let $C \subset \mathbf{P}_{2}$ be a plane curve. As above, we can find a family of projections

$$
h_{t}: C \rightarrow \mathbf{P}_{1}
$$

satisfying

$$
h_{t}^{-1}(y)=h_{0}^{-1}(y) \quad \text { for each } t \in \mathbf{C},|t|<\varepsilon
$$

for some point $y \in \mathbf{P}_{1}$ such that the deformation $h_{t}$ of $h_{0}$ does not come from automorphisms of $\mathbf{P}_{1}$ and each $h_{t}$ is unramified at $h_{t}^{-1}(y)$. Let

$$
f_{t}: C \times \mathbf{P}_{1} \rightarrow \mathbf{P}_{1} \times \mathbf{P}_{1}
$$

be the product

$$
f_{t}:=h_{t} \times \operatorname{Id}_{\mathbf{P}_{1}} .
$$

Then $\left\{f_{t}, t \in \mathbf{C},|t|<\varepsilon\right\}$ is a deformation of $f_{0}$, which does not come from automorphisms of $\mathbf{P}_{1} \times \mathbf{P}_{1}$ and satisfies

$$
f_{t}^{-1}(y \times x)=f_{0}^{-1}(y \times x) \quad \text { for each } t \in \Delta \text { and for each } x \in \mathbf{P}_{1} .
$$

Fix a point $z \in\{y\} \times \mathbf{P}_{1}$. Let

$$
f_{t}^{-1}(z)=\left\{s_{1}, \ldots, s_{r}\right\}
$$

where $r$ is the degree of $C$. Let $M$ be the blow-up of $C \times \mathbf{P}_{1}$ at $s_{1}, \ldots, s_{r}$ and $S$ be the blowup of $\mathbf{P}_{1} \times \mathbf{P}_{1}$ at $z$. Then $f_{t}$ induces a surjective holomorphic map $\psi_{t}: M \rightarrow S$. But it is well known that $S$ is biholomorphic to the blow-up of $\mathbf{P}_{2}$ at two distinct points [2, 4.3]. Thus the family $\left\{\psi_{t}, t \in \mathbf{C},|t|<\varepsilon\right\}$ defines a deformation of the holomorphic map $\psi_{0}$ which does not come from automorphisms of $S$. 
Remark 2.3. - It is natural to ask whether there is a version of Main Theorem when $S$ is a successive blow-up of $\mathbf{P}_{2}$ with the blow-up centers possibly lying on the exceptional divisor of the previous blow-up. For example, one may ask whether an analog of Main Theorem holds if we take sufficiently many successive blow-ups of $\mathbf{P}_{2}$. This is not the case. Example 2.2 can be modified to give an example of deformations of holomorphic maps which does not come from automorphisms of $S$, for $S$ obtained after an arbitrary large number of successive blow-ups of $\mathbf{P}_{2}$. In Example 2.2, pick $\ell>0$ distinct points $Z:=\left\{z_{1}, \ldots, z_{\ell}\right\} \subset\{y\} \times \mathbf{P}_{1}$. Let $M$ be the blow-up of $C \times \mathbf{P}_{1}$ at $f_{t}^{-1}(Z)$ and $S$ be the blow-up of $\mathbf{P}_{1} \times \mathbf{P}_{1}$ at $Z$. Then we get a family of surjective holomorphic maps $\psi_{t}: M \rightarrow S$ which does not come from automorphism of the target. In this case, the image in $\mathbf{P}_{2}$ of the exceptional divisors of the successive blow-ups $S \rightarrow \mathbf{P}_{2}$ has cardinality 2. Note that if the image in $\mathbf{P}_{2}$ of the exceptional divisors of the successive blow-ups $S \rightarrow \mathbf{P}_{2}$ has cardinality $\geqslant 3$, then we can apply Main Theorem to conclude that all deformations of surjective holomorphic maps to $S$ come from automorphisms of $S$.

\section{Infinitesimal automorphisms of a web}

Let $U$ be a 2-dimensional complex manifold and $\mathbf{P} T(U)$ be the projectivization of its holomorphic tangent bundle. Recall that a web on $U$ is a submanifold $W \subset \mathbf{P} T(U)$ with finitely many connected components each of which is biholomorphic to $U$ by the natural projection $\mathbf{P} T(U) \rightarrow U$. If $d$ is the number of the components of $W$, we say that $W$ is a $d$-web. Given an open subset $V \subset U$, the restriction of $W$ to $V$ defines a web on $V$, which we denote by $\left.W\right|_{V}$. Also given an unramified holomorphic map $\psi: U^{\prime} \rightarrow U$, the natural pull-back of $W$ by $d \psi: \mathbf{P} T\left(U^{\prime}\right) \rightarrow \mathbf{P} T(U)$ is a web on $U^{\prime}$, which we denote by $\psi^{-1} W$ and call the pull-back web of $W$. A web $W^{\prime} \subset \mathbf{P} T(U)$ is a sub-web of $W$ if $W^{\prime} \subset W$. A web $W \subset \mathbf{P} T(U)$ on a complex manifold $U$ and a web $W^{\prime} \subset \mathbf{P} T\left(U^{\prime}\right)$ on another complex manifold $U^{\prime}$ are equivalent if there exists a biholomorphic map $\psi: U^{\prime} \rightarrow U$ such that $\psi^{-1} W=W^{\prime}$.

Given a web $W$ on $U$, a holomorphic vector field $v$ on $U$ is an infinitesimal automorphism of $W$ if for any relatively compact domain $U_{0} \subset U$, the 1-parameter family of biholomorphic maps generated by $v$

$$
\left\{\exp (t v): U_{0} \rightarrow U_{t}:=\exp (t v)\left(U_{0}\right), t \in \mathbf{C},|t|<\varepsilon\right\}
$$

for sufficiently small $\varepsilon$ defines an equivalence of webs $\left.W\right|_{U_{0}}$ and $\left.W\right|_{U_{t}}$ for each $t$. Let $v_{1}, \ldots, v_{d}$ be $d$ holomorphic vector fields on $U$ which are pairwise and point-wise independent on $U$. Then their images $\left[v_{1}\right], \ldots,\left[v_{d}\right] \subset \mathbf{P} T(U)$ define a $d$-web, which we call the web defined by the vector fields $v_{1}, \ldots, v_{d}$. It is immediate to see the following two propositions.

PROPOSITION 3.1. - Let $U$ be a 2-dimensional complex manifold with pairwise point-wise independent holomorphic vector fields $v_{1}, \ldots, v_{d}$. A holomorphic vector field $v$ on $U$ is an infinitesimal automorphism of the $d$-web defined by $v_{1}, \ldots, v_{d}$, if and only if for each $i=1, \ldots, d$, there exists a holomorphic function $h_{i}$ on $U$ such that

$$
\left[v, v_{i}\right]=h_{i} \cdot v_{i}
$$

where the bracket denotes the Lie bracket of vector fields.

Proposition 3.2. - Let $W$ be a web on a 2-dimensional complex manifold $U$. Then a holomorphic vector field on $U$ which is an infinitesimal automorphism of $W$, is an infinitesimal automorphism of any sub-web of $W$.

We collect below some explicit calculations regarding the infinitesimal automorphisms of some simple webs on $\mathbf{C}^{2}$. We will fix a standard coordinate system $(x, y)$ on $\mathbf{C}^{2}$.

$4^{e}$ SÉRIE - TOME $40-2007-\mathrm{N}^{\circ} 1$ 
PROPOSITION 3.3. - Let $f(x, y) \frac{\partial}{\partial x}+g(x, y) \frac{\partial}{\partial y}$ be a holomorphic vector field on a domain $U \subset \mathbf{C}^{2}$, which is an infinitesimal automorphism of the web defined by the vector fields $\frac{\partial}{\partial x}$ and $\frac{\partial}{\partial y}$. Then $f(x, y)$ depends only on $x$ and $g(x, y)$ depends only on $y$.

Proof. - This is an easy consequence of Proposition 3.1.

Proposition 3.4. - Fix $a, b \in \mathbf{C}$. Consider a domain $U$ in $\mathbf{C}^{2}$ where the three vector fields

$$
\frac{\partial}{\partial x}, \frac{\partial}{\partial y},(x-a) \frac{\partial}{\partial x}+(y-b) \frac{\partial}{\partial y}
$$

define a 3-web $W$. Let $f(x, y) \frac{\partial}{\partial x}+g(x, y) \frac{\partial}{\partial y}$ be a holomorphic vector field on $U$, which is an infinitesimal automorphism of the web $W$. Then there exist some constants $c_{1}, c_{2}, c_{3} \in \mathbf{C}$ such that

$$
\begin{aligned}
& f(x)=c_{1}(x-a)+c_{2}(x-a) \log (x-a), \\
& g(y)=c_{3}(y-b)+c_{2}(y-b) \log (y-b) .
\end{aligned}
$$

Proof. - By Proposition 3.3, $f(x, y)=f(x)$ and $g(x, y)=g(y)$. Note that

$$
\left[f \frac{\partial}{\partial x}+g \frac{\partial}{\partial y},(x-a) \frac{\partial}{\partial x}+(y-b) \frac{\partial}{\partial y}\right]=\left(f-(x-a) \frac{d f}{d x}\right) \frac{\partial}{\partial x}+\left(g-(y-b) \frac{d g}{d y}\right) \frac{\partial}{\partial y} .
$$

By Proposition 3.1, this should be proportional to $(x-a) \frac{\partial}{\partial x}+(y-b) \frac{\partial}{\partial y}$. Thus

$$
\frac{f-(x-a) \frac{d f}{d x}}{x-a}=\frac{g-(y-b) \frac{d g}{d y}}{y-b} .
$$

Since the right-hand side of $(\dagger)$ depends only on $y$ while the left hand side depends only on $x$, this must be a constant. Thus

$$
\frac{d}{d x}\left(\frac{f}{x-a}-\frac{d f}{d x}\right)=-\frac{f}{(x-a)^{2}}+\frac{1}{x-a} \frac{d f}{d x}-\frac{d^{2} f}{d x^{2}}=0
$$

yielding the ordinary differential equation

$$
(x-a)^{2} \frac{d^{2} f}{d x^{2}}-(x-a) \frac{d f}{d x}+f=0 .
$$

Under the change of variable $x-a=e^{t}$, the equation becomes

$$
\frac{d^{2} f}{d t^{2}}-2 \frac{d f}{d t}+f=0
$$

giving the solution

$$
f=c_{1} e^{t}+c_{2} t e^{t}=c_{1}(x-a)+c_{2}(x-a) \log (x-a)
$$

for some constants $c_{1}, c_{2} \in \mathbf{C}$. Similarly, the equation

$$
\frac{d}{d y}\left(\frac{g}{y-b}-\frac{d g}{d y}\right)=0
$$

ANNALES SCIENTIFIQUES DE L'ÉCOLE NORMALE SUPÉRIEURE 
has the solution

$$
g(y)=c_{3}(y-b)+c_{4}(y-b) \log (y-b)
$$

for some constants $c_{3}, c_{4} \in \mathbf{C}$. Substituting in $(\dagger)$, we see that $c_{2}=c_{4}$.

Proposition 3.5. - Let $a \neq a^{\prime}, b \neq b^{\prime}$ be complex numbers. Consider the web $W$ defined by the following four vector fields on a domain in $\mathbf{C}^{2}$ where they are pairwise and point-wise independent.

$$
\frac{\partial}{\partial x}, \frac{\partial}{\partial y},(x-a) \frac{\partial}{\partial x}+(y-b) \frac{\partial}{\partial y},\left(x-a^{\prime}\right) \frac{\partial}{\partial x}+\left(y-b^{\prime}\right) \frac{\partial}{\partial y}
$$

Then $W$ has no non-zero infinitesimal automorphism.

Proof. - $W$ has two sub-webs $W_{1}$ and $W_{2}, W_{1}$ defined by

$$
\frac{\partial}{\partial x}, \frac{\partial}{\partial y},(x-a) \frac{\partial}{\partial x}+(y-b) \frac{\partial}{\partial y}
$$

and $W_{2}$ defined by

$$
\frac{\partial}{\partial x}, \frac{\partial}{\partial y},\left(x-a^{\prime}\right) \frac{\partial}{\partial x}+\left(y-b^{\prime}\right) \frac{\partial}{\partial y} .
$$

By Propositions 3.2 and 3.4, an infinitesimal automorphism $f(x) \frac{\partial}{\partial x}+g(y) \frac{\partial}{\partial y}$ must satisfy

$$
\begin{aligned}
& f(x)=c_{1}(x-a)+c_{2}(x-a) \log (x-a)=c_{1}^{\prime}\left(x-a^{\prime}\right)+c_{2}^{\prime}\left(x-a^{\prime}\right) \log \left(x-a^{\prime}\right), \\
& g(y)=c_{3}(y-b)+c_{2}(y-b) \log (y-b)=c_{3}^{\prime}\left(y-b^{\prime}\right)+c_{2}^{\prime}\left(y-b^{\prime}\right) \log \left(y-b^{\prime}\right) .
\end{aligned}
$$

It follows that $c_{1}=c_{2}=c_{3}=c_{1}^{\prime}=c_{2}^{\prime}=c_{3}^{\prime}=0$.

Proposition 3.6. - Consider a domain $U$ in $\mathbf{C}^{2}$ where the three vector fields

$$
\frac{\partial}{\partial x}, \frac{\partial}{\partial y}, \frac{\partial}{\partial x}-\frac{\partial}{\partial y}
$$

define a 3-web $W$. Let $f(x) \frac{\partial}{\partial x}+g(y) \frac{\partial}{\partial y}$ be a holomorphic vector field on $U$, which is an infinitesimal automorphism of the web $W$. Then there exist some constants $c_{1}, c_{2}, c_{3} \in \mathbf{C}$ such that

$$
f(x)=c_{1}+c_{2} x, \quad g(y)=c_{3}+c_{2} y .
$$

Proof. - An easy computation using Proposition 3.1 shows that

$$
\frac{d f}{d x}=\frac{d g}{d y}=\text { constant }
$$

Proposition 3.6 follows by setting the constant $c_{2}$.

Proposition 3.7. - Consider a domain $U$ in $\mathbf{C}^{2}$ where the four vector fields

$$
\frac{\partial}{\partial x}, \frac{\partial}{\partial y}, \frac{\partial}{\partial x}-\frac{\partial}{\partial y},(x-a) \frac{\partial}{\partial x}+(y-b) \frac{\partial}{\partial y}
$$

$4^{e}$ SÉRIE - TOME $40-2007-\mathrm{N}^{\circ} 1$ 
define a 4-web $W$. Let $f(x) \frac{\partial}{\partial x}+g(y) \frac{\partial}{\partial y}$ be a holomorphic vector field on $U$, which is an infinitesimal automorphism of the web $W$. Then there exists some constant $c$ such that

$$
f(x)=c(x-a), \quad g(y)=c(y-b) .
$$

Proof. - As in the proof of Proposition 3.5, Proposition 3.2 shows that $f$ and $g$ must be of the form appearing in both Propositions 3.4 and 3.6, from which the result is clear.

\section{Web of fibrations on smooth projective surfaces}

For our purpose, it will be convenient to introduce the notion of a 'web of fibrations'.

Let $S$ be a smooth projective surface. Suppose we are given

(i) a collection of Zariski dense open subsets $U_{1}, \ldots, U_{d}$ of $S$, and

(ii) for each $i=1, \ldots, d$, a smooth quasi-projective curve $C_{i}$ and a surjective proper holomorphic map $f_{i}: U_{i} \rightarrow C_{i}$ such that for each $i \neq j$, the fibers of $f_{i}$ and $f_{j}$ through a general point of $S$ are distinct.

The closure of the kernel of the differential $d f_{i}: T\left(U_{i}\right) \rightarrow T\left(C_{i}\right)$ defines a subvariety $W_{i} \subset$ $\mathbf{P} T(S)$ such that the natural projection $\mathbf{P} T(S) \rightarrow S$ restricted to $W_{i}$ is birational. By the assumption in (ii), $W_{1}, \ldots, W_{d}$ are $d$ distinct (reduced) subvarieties of $\mathbf{P} T(S)$. Let

$$
W:=W_{1} \cup \cdots \cup W_{d} \subset \mathbf{P} T(X) .
$$

A subvariety $W \subset \mathbf{P} T(X)$ with finitely many irreducible components is called a web of fibrations on $S$ if it arises from a collection of surjective proper holomorphic maps $\left\{f_{i}: U_{i} \rightarrow C_{i}\right\}$ as explained above. Strictly speaking, this is not a web in the sense of Section 3. However there is a canonical way of associating a web to a web of fibrations. In fact, given a web of fibrations $W$, there exists a unique maximal Zariski dense open subset in $S$, denoted by $\operatorname{Dom}(W)$, over which $W$ defines a web.

Example 4.1. - Let $P_{1}, \ldots, P_{d}$ be $d$ distinct points on $\mathbf{P}_{2}$. Let $\pi: S \rightarrow \mathbf{P}_{2}$ be the blow-up of the $d$ points and let $E_{i} \subset S$ be the exceptional curve over $P_{i}$. We can identify $E_{i}$ with $\mathbf{P} T_{P_{i}}\left(\mathbf{P}_{2}\right)$ in a canonical manner. Under this identification, let $Q_{i j}, j \neq i$, be the point on $E_{i}$ corresponding to the tangent vector of the line $L_{i j}$ joining $P_{i}$ to $P_{j}$. Let $\tilde{L}_{i j}$ be the strict transform of $L_{i j}$ to $S$. For a fixed choice of $i=1, \ldots, d$, let $U_{i}$ be the open subset of $S$ defined by

$$
U_{i}=S \backslash \bigcup_{i \neq j}\left(\tilde{L}_{i j} \cup E_{j}\right)
$$

Then the pencil of lines passing through $P_{i}$ defines a $\mathbf{P}_{1}$-bundle

$$
f_{i}: U_{i} \rightarrow C_{i}:=E_{i} \backslash\left\{Q_{i j}, i \neq j\right\} .
$$

The web of fibrations defined by $\left\{f_{i}, 1 \leqslant i \leqslant d\right\}$ on $S$ will be called the canonical $d$-web on $S$.

PROPOSITION 4.2. - There exist only countably many webs of fibrations on a given smooth projective surface.

Proof. - Note that each irreducible component of a general fiber of the holomorphic map $f_{i}: U_{i} \rightarrow C_{i}$ defining a web of fibrations is a smooth projective curve with trivial normal bundle. Thus its small deformations in $S$ are irreducible components of fibers of $f_{i}$, too. This means 
that general fibers of $f_{i}$ correspond to a Zariski dense subset of an irreducible component of the Hilbert scheme of curves on $S$. This way, a $d$-web of fibrations on $S$ determines $d$ irreducible components of the Hilbert scheme of curves on $S$. It is clear that these $d$ irreducible components of the Hilbert scheme of curves on $S$ determine the web of fibrations. Thus Proposition 4.2 is a consequence of the countability of the number of irreducible components of the Hilbert scheme.

Proposition 4.3. - Let $\psi: S^{\prime} \rightarrow S$ be a generically finite holomorphic map between two smooth projective surfaces. Let $W \subset \mathbf{P} T(S)$ be a web of fibrations on $S$. Let $U \subset S^{\prime}$ be a Zariski open subset such that $\left.\psi\right|_{U}$ is unramified and $\psi(U) \subset \operatorname{Dom}(W)$. Denote by $\psi^{-1} W \subset \mathbf{P} T\left(S^{\prime}\right)$ the closure of the pull-back of $W$ by $\left.\psi\right|_{U}$. Then $\psi^{-1} W$ is a web of fibrations on $S^{\prime}$.

Proof. - If $\left\{f_{i}: U_{i} \rightarrow C_{i}\right\}$ is the collection of proper holomorphic maps defining $W$ as a web of fibrations, then $\left\{\left.f_{i} \circ \psi\right|_{\psi^{-1}\left(U_{i}\right) \cap U^{\prime}}\right\}$ makes $\psi^{-1} W$ a web of fibrations on $S^{\prime}$.

PROPOSITION 4.4. - Let $W$ be a web of fibrations on a smooth projective surface $S$. Let $v$ be a holomorphic vector field on $S$. Then $\left.v\right|_{\operatorname{Dom}(W)}$ is an infinitesimal automorphism of $W$.

Proof. - Let $\psi: S \rightarrow S$ be a biholomorphic automorphism of $S$. By Proposition 4.3, $\psi$ defines a new web of fibrations $\psi^{-1} W$ on $S$. Thus for a holomorphic vector field $v$ on $S$, the family of biholomorphic automorphisms $\left\{\psi_{t}:=\exp (t v), t \in \mathbf{C},|t|<\varepsilon\right\}$ of $S$ defines a family of webs of fibrations $\psi_{t}^{-1} W$. It is clear that on some analytic open subset they define a holomorphic family of webs parametrized by $t$. By Proposition 4.2, they must be the same web of fibrations, which means that $v$ is an infinitesimal automorphism of the web $W$.

For the next proposition, let us recall the Kodaira-Spencer class of deformations of holomorphic maps (see e.g. [4]). Let

$$
\psi_{t}: S^{\prime} \rightarrow S, \quad t \in \mathbf{C},|t|<\varepsilon
$$

be a deformation of a surjective holomorphic map $\psi_{0}$ between two compact complex manifolds. Then we can define the derivative at $t_{o},\left|t_{o}\right|<\varepsilon$,

$$
\tau_{t_{o}}:=\left.\frac{d}{d t}\right|_{t_{o}} \psi_{t}
$$

as an element of $H^{0}\left(S^{\prime}, \psi_{t_{o}}^{*} T(S)\right) . \tau_{t_{o}}$ is called the Kodaira-Spencer class of the deformation $\psi_{t}$ at $t=t_{o}$. In other words, $H^{0}\left(S^{\prime}, \psi_{t_{o}}^{*} T(S)\right)$ is the space of infinitesimal deformations of $\psi_{t_{o}}$. We know that $H^{0}(S, T(S))$ is the space of infinitesimal automorphisms of $S$. Thus if

$$
H^{0}\left(S^{\prime}, \psi^{*} T(S)\right)=\psi^{*} H^{0}(S, T(S))
$$

for any surjective holomorphic map $\psi: S^{\prime} \rightarrow S$, then all deformations of surjective holomorphic maps from $S^{\prime}$ to $S$ come from automorphisms of $S$.

When $\psi: S^{\prime} \rightarrow S$ is a generically finite holomorphic map, we can regard elements of $H^{0}\left(S^{\prime}, \psi^{*} T(S)\right)$ as multi-valued holomorphic vector fields on $S$. The next result says that Proposition 4.4 can be generalized to multi-valued holomorphic vector fields on $S$, as long as they arise as Kodaira-Spencer classes of deformations of holomorphic maps.

PROPOSITION 4.5. - Let

$$
\psi_{t}: S^{\prime} \rightarrow S, \quad t \in \mathbf{C},|t|<\varepsilon
$$

$4^{e}$ SÉRIE - TOME $40-2007-\mathrm{N}^{\circ} 1$ 
be a deformation of a generically finite holomorphic map $\psi_{0}$ between two smooth projective surfaces. Let $\tau_{0} \in H^{0}\left(S^{\prime}, \psi_{0}^{*} T(S)\right)$ be its Kodaira-Spencer class at $t=0$. Assume that there exists a web $W$ of fibrations on $S$. Choose an analytic open subset $V \subset S^{\prime}$ such that $\psi_{t}(V) \subset$ $\operatorname{Dom}(W)$ and

$$
\left.\psi_{t}\right|_{V}: V \rightarrow \psi_{t}(V)
$$

is biholomorphic for each $|t|<\varepsilon$. Then $\tau_{0} \mid V$, regarded as a holomorphic vector field on $\psi_{0}(V)$, is an infinitesimal automorphism of the web $\left.W\right|_{\psi_{0}(V)}$.

Proof. - By Proposition 4.3, we have a family of webs of fibrations $\psi_{t}^{-1} W$ parametrized by $t$. By Proposition 4.2, they must be identical. Thus

$$
\left.\left(\psi_{t}^{-1} W\right)\right|_{V}=\left.\left(\psi_{0}^{-1} W\right)\right|_{V} \quad \text { for each }|t|<\varepsilon .
$$

This implies the proposition.

\section{Liouville theorem: Blow-up at three collinear points or at four or more points}

Let $W$ be a web of fibrations on a smooth projective surface $S$. We have seen in Proposition 4.4, that a global holomorphic vector field $v$ on $S$ gives an infinitesimal automorphism of $W$. Motivated by the classical Liouville theorem in conformal geometry [1, 15.2], we say that Liouville theorem holds for $W$ if for any connected analytic open subset $V \subset \operatorname{Dom}(W)$ and an infinitesimal automorphism $v_{o}$ of $\left.W\right|_{V}$, there exists a global holomorphic vector field $v \in H^{0}(S, T(S))$ such that $v_{o}=\left.v\right|_{V}$. In particular, if $\left.W\right|_{V}$ has no non-zero infinitesimal automorphism for any analytic open subset $V \subset \operatorname{Dom}(W)$, then Liouville theorem holds trivially.

PROPOSITION 5.1. - Let $S$ be a smooth projective surface with a web of fibrations for which Liouville theorem holds. Let $\psi: S^{\prime} \rightarrow S$ be a generically finite holomorphic map from another smooth projective surface. Then all deformations of $\psi$ come from automorphisms of $S$.

Proof. - Let $\tau \in H^{0}\left(S^{\prime}, \psi^{*} T(S)\right)$ be the Kodaira-Spencer class of a given deformation of $\psi$. It suffices to show that $\tau \in \psi^{*} H^{0}(S, T(S))$. Let $W$ be the web of fibrations. By Proposition 4.5, there exists an analytic open subset $V \subset S$ such that $\tau$ induces an infinitesimal automorphism of $\left.W\right|_{V}$. Since Liouville theorem holds for $W, \tau$ must come from a global holomorphic vector field on $S$.

The following result is a direct consequence of Stein factorization. For details, see [7, 2.2].

Proposition 5.2. - Let $S$ be a smooth projective surface. Assume that for any smooth projective surface $S^{\prime}$ and any generically finite holomorphic map $\psi: S^{\prime} \rightarrow S$, all deformations of $\psi$ come from automorphisms of $S$. Then for any compact complex manifold $M$ and any surjective holomorphic map $\phi: M \rightarrow S$, all deformations of $\phi$ come from automorphisms of $S$.

By Propositions 5.1 and 5.2, the following two propositions prove Main Theorem when $S$ is the blow-up at three collinear points or at four or more points.

PROPOSITION 5.3. - Let $S$ be the blow-up of $\mathbf{P}_{2}$ at 3 collinear points. Then Liouville theorem holds for the canonical 3-web on $S$.

Proof. - Let us use the terminology of Example 4.1. It is easy to see that $H^{0}(S, T(S))$ corresponds to vector fields on $\mathbf{P}_{2}$ vanishing on the line containing the three collinear points. 
In particular, $\operatorname{dim} H^{0}(S, T(S))=3$. Choose a homogeneous coordinate system $\left[z_{0}: z_{1}: z_{2}\right]$ on $\mathbf{P}_{2}$ such that

$$
P_{1}=[0: 1: 0], \quad P_{2}=[0: 0: 1], \quad P_{3}=[0: 1: 1] .
$$

Consider the inhomogeneous coordinate

$$
x=\frac{z_{1}}{z_{0}}, \quad y=\frac{z_{2}}{z_{0}} \quad \text { on the open set } \mathbf{P}_{2} \backslash\left(z_{0}=0\right) \cong \mathbf{C}^{2} .
$$

The web $W$ on $\operatorname{Dom}(W)$ is equivalent to the web in Proposition 3.6 in terms of the above inhomogeneous coordinates via the blow-up $\pi: S \rightarrow \mathbf{P}_{2}$. Consequently, on any analytic open subset $V$ of $\operatorname{Dom}(W)$, the space of infinitesimal automorphisms of $\left.W\right|_{V}$ has dimension 3 by Proposition 3.6. This means that they all come from $H^{0}(S, T(S))$.

Proposition 5.4. - Let $S$ be the blow-up of $\mathbf{P}_{2}$ at $d \geqslant 4$ distinct points. Then Liouville theorem holds for the canonical d-web on $S$.

Proof. - Let us use the terminology of Example 4.1. There are three cases to consider.

(Case 1) All $\left\{P_{1}, \ldots, P_{d}\right\}$ lie on a single line.

(Case 2) $\left\{P_{1}, \ldots, P_{d-1}\right\}$ lie on a single line, but $P_{d}$ are not on the line.

(Case 3) There are four points $P_{1}, \ldots, P_{4}$, no three of which are collinear.

Let us show that these three cases exhaust all possibilities. If $d=4$, then this is clear. So let us assume that $d \geqslant 5$. Assume neither Case 1 nor Case 2 hold. Let $3 \leqslant \ell \leqslant d-2$ be the maximal number of collinear points in $\left\{P_{1}, \ldots, P_{d}\right\}$. We can assume that $\left\{P_{1}, \ldots, P_{\ell}\right\}$ are collinear and lie on a line $L$. Let $L^{\prime}$ be the line joining $P_{d-1}$ and $P_{d}$. By assumption, $L \neq L^{\prime}$. Since $\ell \geqslant 3$, we can choose two points, say, $P_{1}, P_{2}$ on the line $L$ disjoint from $L^{\prime}$. Then no three of $\left\{P_{1}, P_{2}, P_{d-1}, P_{d}\right\}$ can be collinear, and we are in Case 3 .

Now we will prove Proposition 5.4 in each case separately.

(Case 1) In this case, $\operatorname{dim} H^{0}(S, T(S))=3$ and the proof of Proposition 5.2 works verbatim by Proposition 3.2.

(Case 2) In this case, $\operatorname{dim} H^{0}(S, T(S))=1$. We can choose a homogeneous coordinate system with

$$
P_{1}=[0: 1: 0], \quad P_{2}=[0: 0: 1], \quad P_{3}=[0: 1: 1], \quad P_{d}=[1: a: b] .
$$

The web $W$ on $\operatorname{Dom}(W)$ is equivalent to a web which has a sub-web equivalent to the 4-web appeared in Proposition 3.7 in terms of the inhomogeneous coordinate system chosen in the proof of Proposition 5.3. Proposition 3.7 implies that there can be at most 1-dimensional family of local infinitesimal automorphisms of $W$. Since $\operatorname{dim} H^{0}(S, T(S))=1$, they must be those coming from global holomorphic vector fields.

(Case 3) We can choose a homogeneous coordinate system such that

$$
P_{1}=[0: 1: 0], \quad P_{2}=[0: 0: 1], \quad P_{3}=[1: a: b], \quad P_{4}=\left[1: a^{\prime}: b^{\prime}\right]
$$

with $a \neq a^{\prime}, b \neq b^{\prime}$. Then $W$ on $\operatorname{Dom}(W)$ is equivalent to a web which has a subweb equivalent to the 4-web appeared in Proposition 3.5 in terms of the inhomogeneous coordinate system chosen in the proof of Proposition 5.3. Thus by Proposition 3.2 there exists no non-zero infinitesimal automorphism on any analytic open subset $V \subset \operatorname{Dom}(W)$. Thus Liouville theorem holds trivially.

$4^{\text {e }}$ SÉRIE - TOME $40-2007-\mathrm{N}^{\circ} 1$ 


\section{Blow-up at three non-collinear points}

From Section 5, to finish the proof of Main Theorem, it suffices to consider the case when $S$ is the blow-up of three non-collinear points in $\mathbf{P}_{2}$.

Let $P_{1}, P_{2}, P_{3}$ be three non-collinear points in $\mathbf{P}_{2}$ and $\pi: S \rightarrow \mathbf{P}_{2}$ be the blow-up at these three points. It is easy to check that $\operatorname{dim} H^{0}(S, T(S))=2$. Arguing as in the proofs of Propositions 5.3 and 5.4, the canonical 3-web $W$ on $S$ corresponds to the 3-web appeared in Proposition 3.4. By Proposition 3.4, the dimension of the space of infinitesimal automorphisms of $W$ restricted to a domain is 3. It follows that Liouville theorem does not hold for this web. So there is an extra infinitesimal automorphism which does not come from global vector fields on $S$.

Still, it is possible to prove Main Theorem as follows using the fact that the local infinitesimal automorphisms cannot have finite monodromy.

Proof of Main Theorem. - By Proposition 5.2, we can assume that $S^{\prime}$ is a smooth projective surface. For a generically surjective holomorphic map $\psi: S^{\prime} \rightarrow S$, the Kodaira-Spencer class $\tau$ of any deformation of $\psi$ defines a multi-valued holomorphic vector field on $S$ which is locally an infinitesimal automorphism of $W$ by Proposition 4.5. Note that $\tau$ has finitely many multivalues with the number of different values bounded by the degree of the map $\psi$. But since $W$ is equivalent to the web appeared in Proposition 3.4, if a multi-valued vector field is locally an infinitesimal automorphism of $W$, either it is univalent or it has infinitely many multi-values, depending on whether the coefficient $c_{2}$ in Proposition 3.4 vanishes or not. Thus $\tau$ must be a univalent holomorphic vector field on $S$. It follows that any deformation of $\psi$ comes from automorphisms of $S$. This finishes the proof of Main Theorem.

\section{REFERENCES}

[1] Dubrovin B.A., Fomenko A.T., Novikov S.P., Modern Geometry I, Grad. Texts in Math., vol. 93, Springer-Verlag, Berlin/New York, 1984.

[2] Griffiths P., HARRIS J., Principles of Algebraic Geometry, John Wiley and Sons, New York, 1978.

[3] HÉnAut A., Analytic web geometry, in: Web Theory and Related Topics, Toulouse, 1996, World Sci. Publishing, River Edge, NJ, 2001, pp. 6-47.

[4] HoriKawa E., On deformations of holomorphic maps I, J. Math. Soc. Japan 25 (1973) 372-396.

[5] Hwang J.-M., Rigidity of surjective holomorphic maps to Calabi-Yau manifolds, Math. Zeit. 249 (2005) 767-772.

[6] Hwang J.-M., Deformation of holomorphic maps onto Fano manifolds of second and fourth Betti numbers 1, Ann. Inst. Fourier, submitted for publication.

[7] Hwang J.-M., Kebekus S., Peternell T., Holomorphic maps onto varieties of non-negative Kodaira dimension, J. Alg. Geom. 15 (2006) 551-561.

[8] Hwang J.-M., MoK N., Finite morphisms onto Fano manifolds of Picard number 1 which have rational curves with trivial normal bundles, J. Alg. Geom. 12 (2003) 627-651.

[9] Hwang J.-M., MoK N., Birationality of the tangent map for minimal rational curves, in: Special issue dedicated to Yum-Tong Siu, Asian J. Math. 8 (2004) 51-64.

(Manuscrit reçu le 7 juin 2006;

accepté, après révision, le 12 décembre 2006.)

Jun-Muk Hwang

Korea Institute for Advanced Study,

207-43 Cheongryangri-dong,

Seoul, 130-722, South Korea

E-mail: jmhwang@kias.re.kr 